\title{
Editorial
}

\section{Challenges and Opportunities of Network Virtualization over Wireless Mobile Networks}

\author{
Xiaohong Jiang, ${ }^{1}$ Gabriel-Miro Muntean, ${ }^{2}$ George Ghinea, ${ }^{3}$ and Changqiao $\mathrm{Xu}^{4}$ \\ ${ }^{1}$ Future University Hakodate, Hakodate, Japan \\ ${ }^{2}$ Dublin City University, Dublin, Ireland \\ ${ }^{3}$ Brunel University London, Uxbridge, UK \\ ${ }^{4}$ Beijing University of Posts and Telecommunications, Beijing, China \\ Correspondence should be addressed to Xiaohong Jiang; jiang@fun.ac.jp
}

Received 6 February 2017; Accepted 6 February 2017; Published 26 March 2017

Copyright (c) 2017 Xiaohong Jiang et al. This is an open access article distributed under the Creative Commons Attribution License, which permits unrestricted use, distribution, and reproduction in any medium, provided the original work is properly cited.

Enabled by the rising of mobile communication technologies, wireless mobile networks have become a crucial component of numerous Internet applications such as mobile sensing, video streaming, data dissemination, and social networks. With this growing trend, wireless mobile networks have the potential to reshape the way in which we lead our daily life. Large efforts have been made by the researchers recently, yet problems such as energy consumption, network resource allocation, and efficient data dissemination and transmission remain unsolved in wireless mobile networks.

Network virtualization as a concept enables abstraction and sharing of infrastructure and radio spectrum resource has become very popular in future communication technologies, such as $5 \mathrm{G}$ wireless network and cloud radio access networks (Cloud-RAN). With virtualization, the overall cost of equipment and management can be significantly reduced due to the increased network resource utilization. Additionally, the decoupling of network functionality from infrastructure makes it easier to migrate and test newer network architectures such as software-defined networks (SDN), contentcentric networks $(\mathrm{CCN})$, and emerging service and applications. Although network virtualization has been proved as a promising technology for future Internet, despite the opportunities brought by the network virtualization, several significant research challenges need to be addressed before the wide deployment mobile wireless network virtualization, including resource allocation and management, mobility, QoE guarantee of end users, and the future trend of wireless mobile network virtualization.
After thorough and meticulous reviews, we have selected 9 papers for this special issue.

Firstly, there are two articles dealing with the problem of dynamic resource caching for wireless mobile networks by making use of network virtualization technologies. In "A Novel Video Sharing Solution Based on Demand-Aware Resource Caching Optimization in Wireless Mobile Networks," S. Jia et al. propose a novel video sharing solution based on Demand-aware Resource Caching Optimization (VDRCO), in order to reduce network load and ensure user quality of experience (QoE). L. Zhong et al., in "A Novel Contribution-Aware Neighbor-Assist Video Delivery Solution over Mobile Content-Centric Networks," propose a novel contribution-aware neighbor-assist video delivery solution over mobile content-centric network (CNVD). CNVD designs the estimation methods of interest level and lookup capacity of nodes for video content, which enables the nodes to decide whether to build neighbor relationship with other nodes.

Leveraging network virtualization technologies, the paper by R. Zhang and S. Xiong entitled "A Novel Mobile Video Community Discovery Scheme Using OntologyBased Semantical Interest Capture" proposes a novel mobile Video Community discovery scheme using ontology based semantical interest capture (VCOSI) to construct scalable and resilient node communities, which promotes video sharing capacity of video systems with the flexible and economic community maintenance. 
The issue of dense wireless spaces for networking is addressed in the paper by M. Zhanikeev entitled "Improving Performance in Dense Wireless Spaces by Controlling Bulk Traffic." The author proposes a simple method called Bulk-nPick which minimizes the number of prolonged concurrent sessions by separating bulk from sync traffic. Moreover, the real-life experiments with various counts of wireless devices, bulk sizes, and levels of sync intensity are performed for realistic performance analysis.

The secure service composition for mobile clouds is addressed in the paper by N. Xi et al. entitled "Distributed Secure Service Composition with Declassification in Mobile Clouds." The authors propose a declassification mechanism for secure service composition based on cryptographic operations and information flow security requirements.

Accurate location service is also an important issue on wireless mobile networks. The paper by Q. Liu et al. entitled "A Wireless Location System in LTE Networks" proposes a new LTE location system in Centralized Radio Access Network (C-RAN), which makes channel and location measurement more available, allocation of baseband processing resources more flexible, and location service capability opening. In "GuideLoc: UAV-Assisted Multitarget Localization System for Disaster Rescue," A. Wang et al. propose GuideLoc, a highly efficient aerial wireless localization system, which uses the target guiding technology based on region division. By utilizing GuideLoc, the efficiency of search and rescue tasks can be improved.

Concerning mathematical modeling and network emulation, two papers focus on the dynamic topologies for virtualized sensor networks and emulation platform for delay tolerant networks, respectively. In "Diffusion Strategies for Distributed Kalman Filter with Dynamic Topologies in Virtualized Sensor Networks," S. Yang et al. propose a diffusion Kalman filter algorithm with dynamic topologies (DKFdt) to guarantee the accuracy of estimate values by sensors in physical layer of VSNs. The paper by H. Li et al. entitled "EmuStack: An OpenStack-Based DTN Network Emulation Platform (Extended Version)" proposes a largescale real-time emulation platform named EmuStack, which aims at empowering network emulation to become as simple as network simulation.

\section{Acknowledgments}

We would like to thank all the reviewers for their hard work and for all their suggestions and comments, leading to improvements in the accepted papers. By compiling these papers, we hope that it will represent a useful starting point and stimulus for further research on network virtualization technologies in wireless mobile networks. We would like to take this opportunity to thank all authors for submitting their works to this special issue.

Xiaohong Jiang

Gabriel-Miro Muntean

George Ghinea

Changqiao Xu 

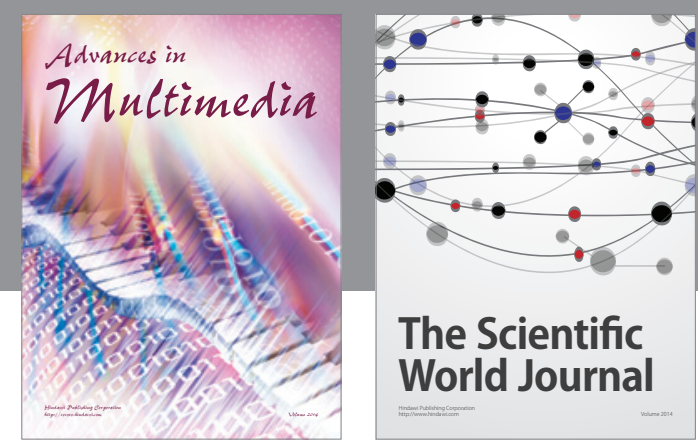

The Scientific World Journal
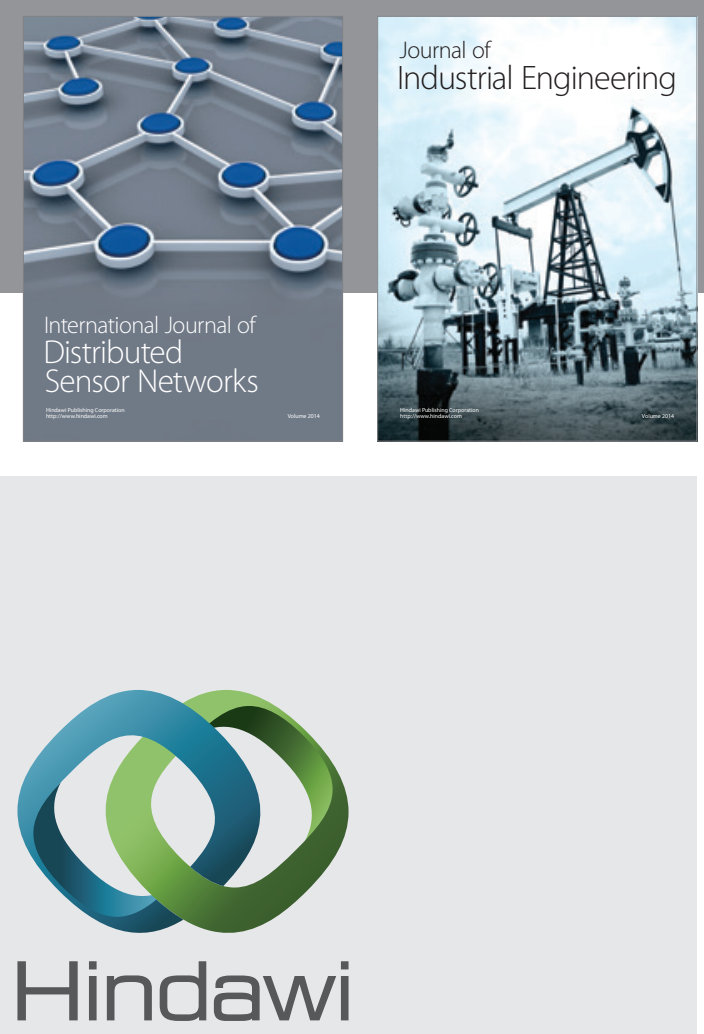

Submit your manuscripts at

https://www.hindawi.com

\section{Computer Networks} and Communications
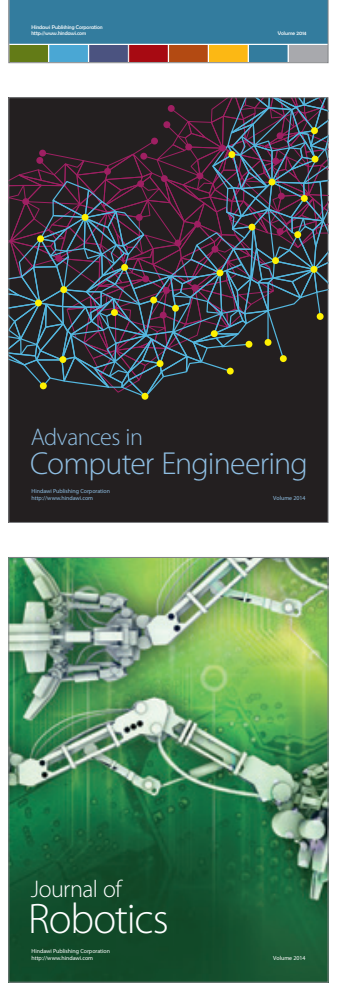
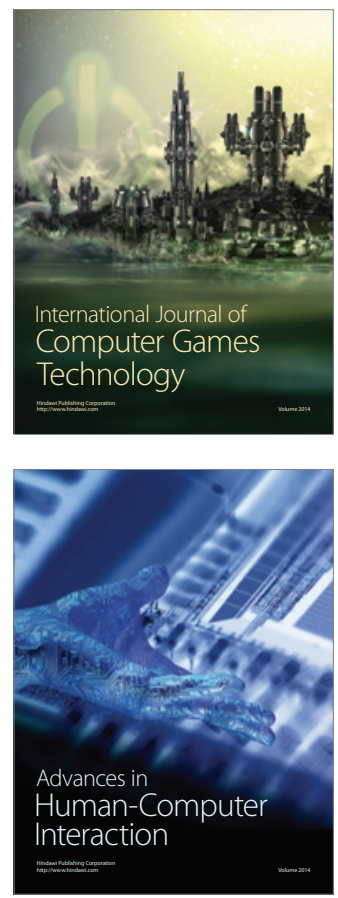
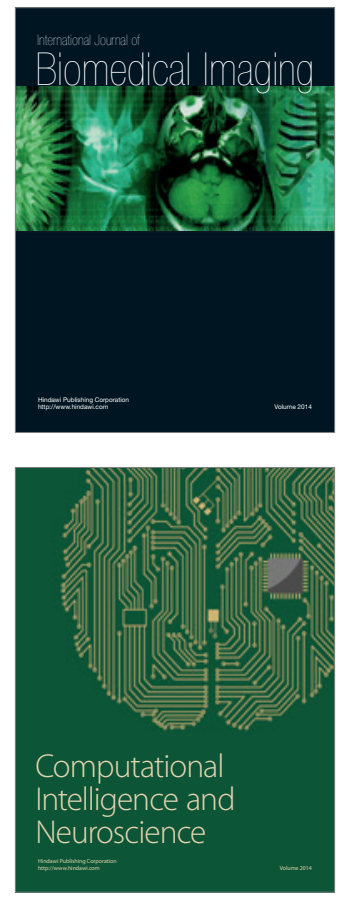
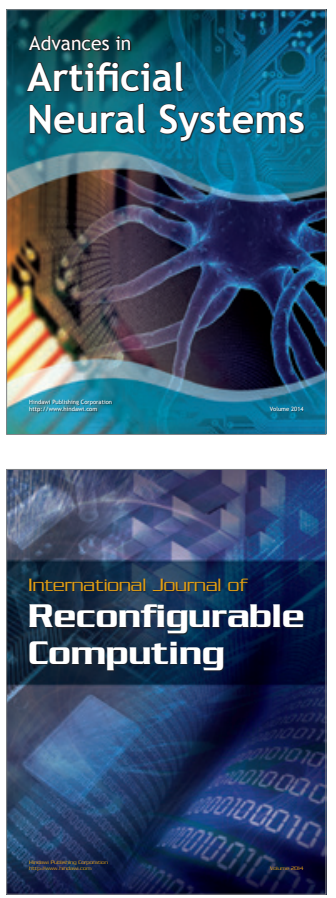
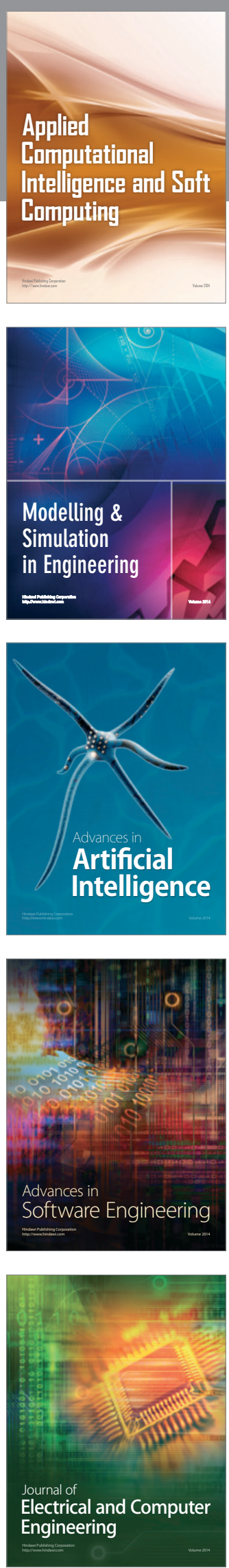